\title{
Percepción de los estudiantes de posgrado en gerontología sobre el envejecimiento
}

Eloy López Meneses

Universidad Pablo de Olavide (España)

José Luis Sarasola Sánchez-Serrano

Universidad Pablo de Olavide (España)

Alicia Jaén Martínez

Universidad Pablo de Olavide (España)

José Gómez Galán

Universidad Metropolitana (Puerto Rico) 



\title{
Percepción de los estudiantes de posgrado en gerontología sobre el envejecimiento
}

\section{Gerontology graduate students' perception of ageing}

\author{
Eloy López Meneses \\ Universidad Pablo de Olavide (España) \\ elopmen@upo.es
}

José Luis Sarasola Sánchez-Serrano

Universidad Pablo de Olavide (España)

jlsarsan@upo.es

\author{
Alicia Jaén Martínez \\ Universidad Pablo de Olavide (España) \\ ajaemar@upo.es \\ José Gómez Galán \\ Universidad Metropolitana (Puerto Rico) \\ jgomez@suagm.edu
}

Fecha de recepción: 01 de diciembre de 2017

Fecha de aceptación: 11 de diciembre de 2018

Financiación: Línea de trabajo iniciada en la investigación "Innovación docente 2.0 con Tecnologías de la Información y la Comunicación (TIC) en el Espacio Europeo de Educación Superior", situada en el marco de la Acción 2 de Proyectos de Innovación y Desarrollo Docente subvencionados por el Vicerrectorado de Docencia y Convergencia Europea de la Universidad Pablo de Olavide (Sevilla, España)

\section{Resumen}

Hoy en día, por primera vez en la historia, la mayoría de las personas puede aspirar a vivir más allá de los 60 años. En los países de ingresos bajos y medianos, esto se debe en gran parte a la notable reducción de la mortalidad en las primeras etapas de la vida, sobre todo durante la infancia y el nacimiento, y de la mortalidad por enfermedades infecciosas. En los países de ingresos altos, el aumento sostenido de la esperanza de vida actualmente se debe sobre todo al descenso de la mortalidad entre las personas mayores. En este sentido, el número de habitantes en América Latina ascenderá hacia mediados de 2016 a 625 millones de personas, seis millones más de los que se estima que había a mitad de 2015, y se calcula que alcanzará 680 millones en 2025 y 779 millones en 2050, según las últimas proyecciones 
de población de la Comisión Económica para América Latina y el Caribe. Y en la Unión Europea se prevé que la población aumentará de 507 millones en 2013 hasta el año 2050 en casi un 5\%, cuando llegará a su máximo (en 526 millones). En consonancia con este nuevo planteamiento global, se realizó un estudio diacrónico con los estudiantes de posgrado que cursan el Máster Oficial de Gerontología, Dirección y Gestión de Centros Gerontológicos de la Universidad Pablo de Olavide, durante los cursos académicos 2012-2013 a 2015-16. A través de una investigación descriptiva y de métodos mixtos -con técnicas y procesos de reducción de datos, codificación, interpretación e inferencia, etc.-, se analizó el diseño y elaboración por parte del alumnado de nubes de ideas desarrolladas con software social sobre las concepciones relacionadas con el proceso de envejecimiento a partir de los contenidos impartidos en el módulo teórico y de prácticas en centros gerontológicos.

Palabras clave: Personas Mayores; Envejecimiento; Nube de Conceptos; Mapa conceptual; Experiencia posgrado.

\begin{abstract}
Nowadays, for the first time in history, most people can expect to live beyond 60 years. In low- and middle-income countries, this is largely due to the significant reduction of mortality in the early stages of life, especially during childhood and birth, and from infectious diseases. In high-income countries, the sustained increase in life expectancy today is owing to the decline of mortality among the elderly. In this regard, the number of inhabitants in Latin America will be rising by mid-2016 to 625 million people; six million more than there would be estimated by mid-2015. According to the latest population projections of the United Nations Economic Commission for Latin America and the Caribbean, 680 million people by 2025 and 779 million in 2050 will be reached. And in the European Union, a population increase of 5 per cent is expected: from 507 million by 2013, until 526 million by 2050 , achieving the maximum. In line with this new global approach, a diachronic study with graduate students attending the Official Master's Degree in Gerontology, Direction and Management of Gerontology Centres at Pablo de Olavide University, during 2012-2013 to 2015-2016 academic years, was realized. Through a descriptive and mixed methods research -with techniques and processes of data reduction, coding, interpretation and inference, etc.-, the students' design and elaboration of clouds of ideas -developed with social software- about concepts related to the ageing process, from the contents taught in the theoretical module and the internship in different gerontology centres, were analysed.
\end{abstract}

Keywords: Elderly People; Ageing; Concept Cloud; Concept Map; Graduate Experience.

Para citar este artículo: López Meneses, E.; Sarasola Sánchez-Serrano, J. L.; Jaén Martínez, A. y Gómez Galán, J. (2019). Percepción de los estudiantes de posgrado en gerontología sobre el envejecimiento. Revista de humanidades, n. 36, pp. 155-180. ISBN 1130-5029 (ISSN-e 2340-8995).

Sumario: 1. Introducción. 2. Fundamentos teóricos. 3. Contex to de la investigación. 4. Objetivos. 5. Metodología de la investigación. 6. Resultados. 7. Conclusiones. 8. Referencias bibliográficas. 


\section{INTRODUCGIÓN}

Hoy en día, por primera vez en la historia, la mayoría de las personas puede aspirar a vivir más allá de los 60 años. En los países de ingresos bajos y medianos, esto se debe en gran parte a la notable reducción de la mortalidad en las primeras etapas de la vida, sobre todo durante la infancia y el nacimiento, y de la mortalidad por enfermedades infecciosas. En los países de ingresos altos, el aumento sostenido de la esperanza de vida actualmente se debe sobre todo al descenso de la mortalidad entre las personas mayores (Organización Mundial de la Salud, 2015). En este sentido, el número de habitantes en América Latina ascenderá hacia mediados de 2016 a 625 millones de personas, seis millones más de los que se estima que había a mitad de 2015, y se calcula que alcanzará 680 millones en 2025 y 779 millones en 2050, según las últimas proyecciones de población de la Comisión Económica para América Latina y el Caribe (VV. AA, 2015). Sirva a modo de ejemplo, los indicadores del proceso de envejecimiento de la población de Venezuela, período 2015-2100 (tabla 1).

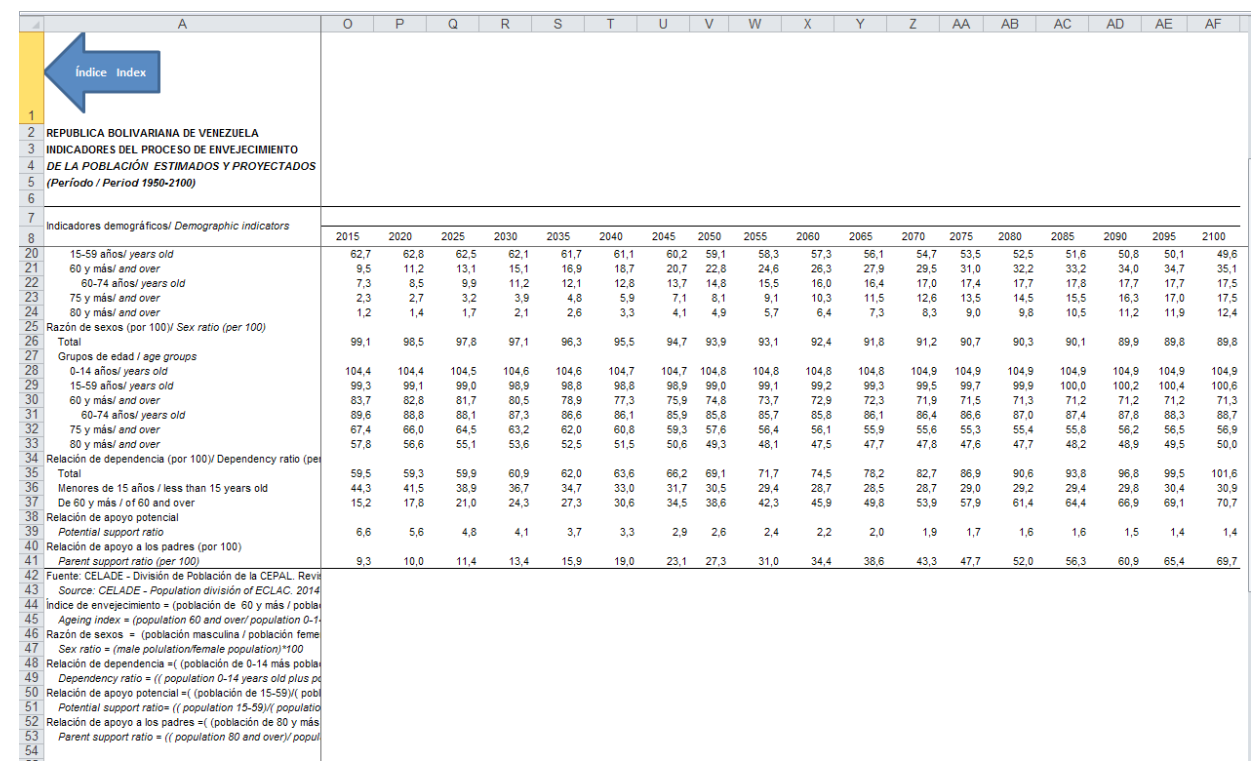

Tabla 1. Indicadores del proceso de envejecimiento de la población de Venezuela. CELADE - División de Población de la GEPAL (VV. AA, 2015).

Asimismo, en la Unión Europea se prevé que la población aumentará de 507 millones en 2013 hasta el año 2050 en casi un 5\%, cuando llegará a su máximo (en 526 millones). En concordancia con Thumala, Arnold y Urquiza (2010), se puede entender que este sostenido y reciente aumento de la población de adultos mayores es un fenómeno a escala planetaria. 
En este sentido, el presente estudio diacrónico derivado de la línea de trabajo iniciada en la investigación "Innovación docente 2.0 con Tecnologías de la Información y la Comunicación (Tic) en el Espacio Europeo de Educación Superior", situada en el marco de la Acción 2 de Proyectos de Innovación y Desarrollo Docente subvencionados por el Vicerrectorado de Docencia y Convergencia Europea de la Universidad Pablo de Olavide (Sevilla), se describe un estudio diacrónico con los estudiantes de posgrado que cursan el Máster Oficial de Gerontología, Dirección y Gestión de Centros Gerontológicos de la Universidad Pablo de Olavide, durante los cursos académicos 2012/13, 2013/14,2014/15 y 2015/16. A través de una metodología de carácter descriptivo y de corte cualitativo, se analizó el diseño y elaboración por parte del alumnado de nubes de ideas desarrolladas con el software social Wordle y Tagxedo sobre las concepciones relacionadas con el proceso de envejecimiento a partir de los contenidos impartidos en el módulo teórico y de prácticas en centros gerontológicos.

El procedimiento metodológico seguido, como se desarrollará extensivamente más adelante, consistió en analizar en el estudiantado el concepto de "vejez" utilizando como base distintas nubes de conceptos - o words clouds en inglés- sobre los aspectos relacionados con el mismo. Esto puede realizarse con programas informáticos específicos dentro de lo que se define como software social. Las herramientas empleadas permitieron crear nubes de palabras interactivas, personalizadas y configurables que pudieron descargarse en varios formatos y compartirlos en red. En función de la plataforma en la que se empleen estas herramientas puede ser necesario tener instalado el plugin Silverlight. El formato web permite obtener el código necesario para poder incrustar esa nube en cualquier espacio de contenidos. En todos los casos la nube de palabras es, en esencia, un conjunto de palabras que forman una representación por medio de la imagen que las engloba, y que facilita enormemente el análisis de conceptos.

En este contexto, la vejez, como un estado personal, hace referencia a la última etapa de la vida y se define como "persona de mucha edad". El término "vejez", junto con algunos derivados de este, como "viejo" y "envejecimiento", aluden a una condición temporal y a la consecuencia del tiempo en la persona, o lo que es lo mismo, a la edad (Fernández-Ballesteros, 2000; Rodríguez-Rodríguez, et al., 2012).

El envejecimiento de la población en la Unión Europea es un hecho constatado, cada día hay más personas mayores (Agudo, Pascual y Fombona, 2012). En este sentido, las previsiones de las Naciones Unidas estiman que, en el año 2050, España estará entre los diez países más viejos del mundo con un 38,3\% del total, según el informe "Aging in the Twenty-First Century: A Celebration and A Challenge" (2012). En concordancia con Montero (2005), si históricamente el siglo XIX se caracterizó por un «derroche de vida» (alta natalidad y alta mortalidad), el siglo XX ha supuesto el periodo de finalización de la transición demográfica, la revolución de la longevidad y el envejecimiento. 
Tradicionalmente la enseñanza universitaria se ha fundamentado en un modelo metodológico centrado en el docente, con énfasis en la transmisión de contenidos y su reproducción por los alumnos, la lección magistral y el trabajo individual. Enseñar a través de las Tecnologías de la Información y la Comunicación (TIC) demanda una serie de cambios que generan una ruptura de este modelo, al mismo tiempo que suponen un avance hacia la calidad de la educación universitaria (López Meneses y Gómez Galán, 2010; Aguaded, López Meneses y Alonso, 2010; Gómez Galán y Lacerda, 2012).

En la línea discursiva de Martínez-Miguélez (2011), la educación moderna debe hacer un cambio de paradigma, es decir, un cambio de la idea central que la define. El nuevo profesor será un animador, un experto en la mayéutica, que buscará situaciones problemáticas de la vida y las propondrá al alumnado, situaciones que tengan en cuenta todas las dimensiones del ser humano: inteligencia, sensibilidad y cuerpo, es decir, en el nuevo contexto europeo universitario es necesario un cambio metodológico y evaluador orientado (Aguaded, López Meneses y Jaén, 2013) que potencie la formación integral de los estudiantes a través del desarrollo de sus competencias sociales, intelectuales y tecnológicas en el contexto universitario, es decir, en palabras de Cróquer y Paz (2013) en ese entorno circunscrito por una misión y una visión específicas.

\section{FUNDAMENTOS TEÓRICOS}

El envejecimiento es un proceso que se vive desde el nacimiento y se caracteriza por diferentes cambios en niveles físicos, mentales, individuales y colectivos. Estos cambios definen a las personas cuando ya están mayores, pero se debe ver como un proceso natural, inevitable y no necesariamente ligado a estereotipos; como una etapa de la vida apta para "crecer y continuar aprendiendo" (OPS, 2002).

Básicamente podemos hablar de cuatro tipos de estereotipos: el cronológico, asentado en el criterio de normas de edad equipara el envejecimiento con el número de años vividos, a pesar de que muchas personas mayores se encuentran aceptablemente íntegras tanto física como psíquicamente mientras que sujetos de menor edad soportan evidentes deterioros; el biológico, la equiparación de vejez y persona mayor con senilidad, que contribuye injustamente a concebir la vejez como etapa vital cargada de enfermedades; el psicológico, que considera la vejez como deterioro y declive de los recursos psicológicos sensoriales, atencionales, memorísticos, cognoscitivos, aptitudinales, etc., potenciando el mito de la vejez como etapa de escasa o nula creatividad, de aislamiento intimista, de ansiedad y depresiones, etc.; y el sociológico, que ha incidido tradicionalmente en las connotaciones de inutilidad, aislamiento, improductividad y desvinculación de la persona mayor respecto a los intereses sociales y relacionales comunitarios (Carbajo, 2009). 
Por su parte, Montañés y Latorre (2004) indican que el envejecimiento es un proceso adaptativo, lento y continuo, que supone una serie de modificaciones en todos los ámbitos, y en el que intervienen numerosos factores biológicos, psicológicos y sociales. El envejecimiento de la población es el resultado de importantes cambios demográficos desarrollados en los últimos decenios en España. Desde finales de los años setenta del pasado siglo, se ha producido una fuerte caída de la fecundidad que ha elevado el peso porcentual de las personas mayores. Asimismo, el incremento de la esperanza de vida ha supuesto un aumento del número de supervivientes de cada cohorte a lo largo de todas edades del curso de la vida más allá de los 65 años (Yanguas, 2006; VV. AA, 2011).

En este sentido, la cuestión del envejecimiento de la población, en sus múltiples y diversas dimensiones, es un tema de enorme interés; no solo científico sino, sobre todo, social. Las publicaciones científicas y las reuniones específicas ponen de manifiesto su pujanza en la investigación básica y aplicada. Los foros de usuarios y las reuniones de personas mayores, prueban la relevancia que subyace al envejecimiento como fenómeno con pujanza social (Rodríguez, Rodríguez, Sancho y Díaz, 2012).

Asimismo, el envejecimiento de la población en la Unión Europea es un hecho constatado, cada día hay más personas mayores (Agudo, Pascual y Fombona, 2012). Si tomamos como referencia el informe del Instituto de Mayores y Servicios Sociales de 2012 "Portal Mayores" (Abellán y Ayala, 2012), el número de personas de 65 y más años era superior a 8,2 millones, lo que representa el 17,4\% de la población. Las previsiones de las Naciones Unidas estiman que, en el año 2050, España estará entre los diez países más viejos del mundo con un 38,3\% del total, según el informe "Aging in the Twenty-First Century: A Celebration and a Challenge." Mikulak (2012).

El concepto ha ido evolucionando, como señala Zamarrón (2013), desde la definición de la Organización Mundial de la Salud (OMS) de 1990, del envejecimiento saludable (centrado en la salud), hacia un modelo mucho más integrador, como el de envejecimiento activo, es decir, "el proceso de optimización de oportunidades para la salud, la participación y la seguridad que permitan garantizar la calidad de vida de las personas a medida que envejecen" (Organización Mundial de la Salud, 2002, p. 12).

Asu vez, el modelo de envejecimiento activo asume un enfoque intergeneracional que reconoce la importancia de las relaciones familiares y el apoyo prestado entre miembros de distintas generaciones (Sáez, 2002; Fernández-Ballesteros, et al., 2010).

La promoción del envejecimiento activo implica la optimización de todas esas condiciones a través de intervenciones biomédicas, físicas, psicológicas, socioambientales y, sobre todo, educativas. Hemos de subrayar así mismo que la promoción del envejecimiento activo supone prevenir la enfermedad y la discapacidad y mejorar el bienestar y la calidad de vida en la vejez (Fernández Ballesteros, 2009; Gallardo, Peñacoba y Martínez, 2009, p. 67).

En concordancia con Amador-Muñoz y Esteban-Ibañez (2015) los avances en las ciencias de la salud, incluida la nutrición humana, han permitido prolongar 
de manera espectacular la esperanza de vida, aunque en la actualidad no nos conformamos tan sólo con vivir más tiempo, sino que perseguimos mantener, a esa edad, un buen estado de salud y calidad de vida. En este sentido, es preciso erradicar concepciones erróneas sobre la vejez y distinguir los cambios inherentes a este periodo vital de los trastornos o alteraciones que provoca la enfermedad. El declive biológico que conlleva el proceso de envejecimiento no tiene por qué ir acompañado irremediablemente de un deterioro orgánico, puesto que envejecimiento y enfermedad no son términos equivalentes. Si bien es cierto que en la última etapa de la vida se da una probabilidad mayor de padecer patologías, de este hecho solo se deriva la trascendencia de adoptar medidas que procuren un adecuado mantenimiento de la salud de los mayores, entendida esta en su más amplio sentido. Atendiendo a la dimensión bio-psicosocial de la salud, su cuidado durante el envejecimiento debe comprender una actividad física regular, una alimentación equilibrada y un trabajo cognitivo mantenido en los aspectos físicos y psicológicos. Las relaciones familiares y la posibilidad de participación comunitaria son elementos que inciden asimismo en el nivel de salud respecto al plano social (Rojo-Pérez et al., 2009).

Que las personas mayores tengan oportunidad de desarrollar apropiadamente sus capacidades personales a través de la educación, contribuye no solo a prevenir situaciones de dependencia, sino que faculta para el desarrollo de un envejecimiento más pleno. Las intervenciones de Educación para la Salud dirigidas a este colectivo proporcionan un conjunto de herramientas que acrecienta la capacidad personal y favorece la adopción de actitudes críticas sobre los determinantes que inciden en la evolución del proceso de envejecimiento como construcción sociocultural (Beauvoir, 1983). La vivencia emocional del proceso de envejecimiento tiene una influencia fundamental en el desarrollo del mismo, pues, según señala Bazo (1990), una persona se considera vieja cuando la sociedad así la califica.

Son variados los estudios, tanto en el ámbito de América Latina y España (Gairín, Rodríguez-Gómez, Armengol y del Arco Bravo, 2014; González Raimundí, 2015 y 2016; García Rodríguez, Meseguer Martínez, González Losada y Pozo Muñoz, 2016) como en un contexto general internacional (Schuetze y Slowey, 2002; Kasworm, 2012; Cook, 2013; Markle, 2015) que han demostrado los beneficios que la participación de las personas mayores en los contextos universitarios, a través de diferentes programas formativos dirigidos especialmente a este sector de edad o bien articulándose en diferentes titulaciones universitarias

Por último, en el contexto formativo, siguiendo a Martín García y Requejo (2005), una de las intenciones de una acción educativa en la vejez consiste, precisamente, en alcanzar y/o mejorar el bienestar personal y la calidad de vida de todas aquellas personas que viven su proceso de envejecimiento, la educación en la vejez propone un tipo de intervención mediante la cual se trata de descubrir el mejor modo de ayudar a las personas a reconocer las posibilidades que las diferentes fases y momentos de la vejez ofrecen, tratando de favorecer un mejor ajuste y una mayor satisfacción vital (Elosúa, 2002; Escarbajal de Haro, 2003). 
El análisis del proceso de envejecimiento desde una perspectiva educativa es un aspecto necesario y deseable en el actual contexto del Espacio Europeo de Educación Superior; así como el fomento del trabajo colaborativo y con herramientas digitales con integración en la actual dinámica de la Sociedad de la Información y la Comunicación.

\section{CONTEXTO DE LA INVESTIGACIÓN}

La presente experiencia de posgrado se desarrolla en el Máster universitario en Gerontología y Dirección y Gestión de Centros Gerontológicos perteneciente al Centro de Estudios de Posgrado de la Universidad Pablo de Olavide (UPO) de Sevilla.

Es un Máster semipresencial con docencia virtual los lunes a través de la plataforma de aprendizaje Blackboard (UPO); y martes, miércoles y jueves docencia presencial en la Universidad Pablo de Olavide con horario de 17.00 a 21.00. Consta de 60 créditos de docencia y permite el acceso al programa de Doctorado de Ciencias Sociales de la Universidad Pablo de Olavide al estudiantado de Grado y Licenciados. Su URL es http://www.upo.es/postgrado/Master-Oficial-Gerontologia-y-Direcciony-Gestion-de-Centros-Gerontologicos?opcion= $($ figura 1$)$

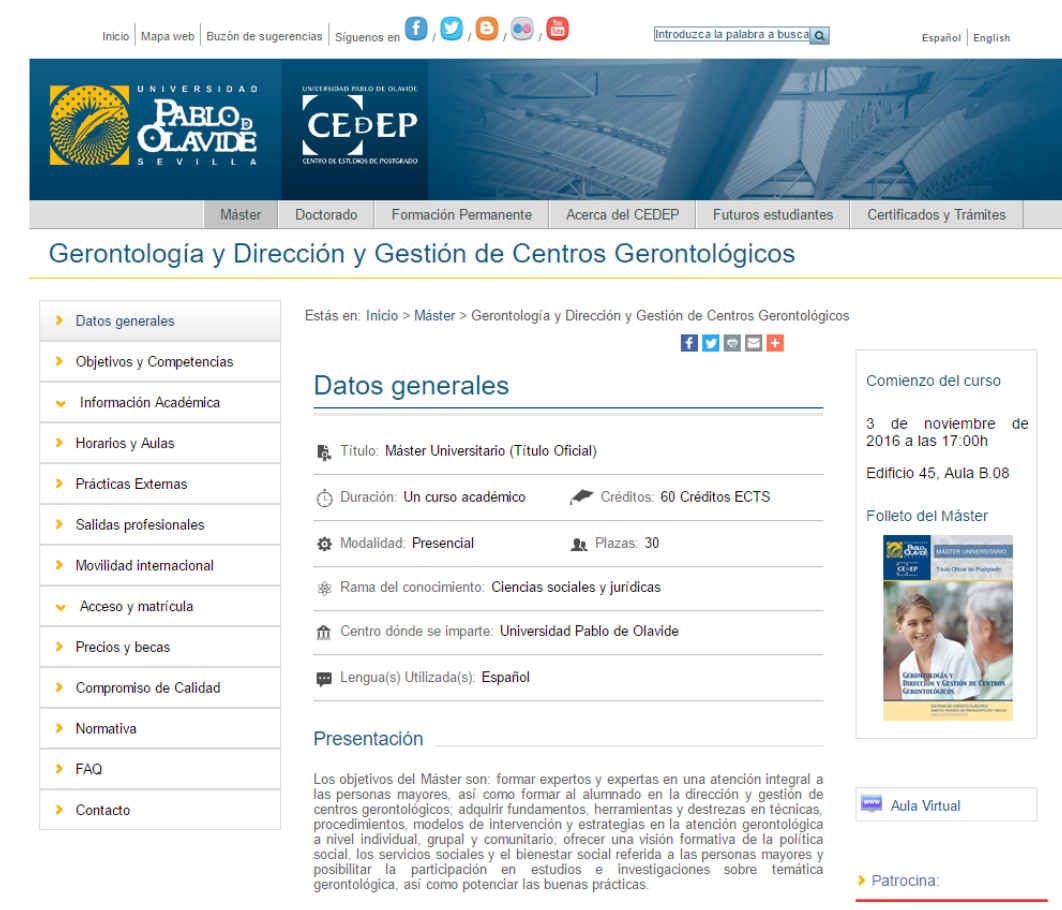

Figura 1. Espacio virtual de Máster de Gerontología y Dirección y Gestión de Centros Gerontológicos. 
Referente a los contenidos del Máster Oficial se concretan en los siguientes módulos formativos: (1) Módulo de Ciencias Gerontológicas; (2) Módulo de dirección, Organización y Gestión de Centros Gerontológicos; (3) Módulo Proyecto de Investigación y Prácticas en Centros Gerontológicos; y (4) Talleres y Seminarios formativos.

La investigación describe un estudio con nubes de conceptos sobre las concepciones de posgrado acerca del envejecimiento. El desarrollo de la misma se plantea comouna continuación de la experiencia de carácterinnovador que se desarrolló de modo inicial durante el curso académico 2012/13 con estudiantes pertenecientes al Máster de Gerontología y Dirección y Gestión de Centros Gerontológicos de la Universidad Pablo de Olavide. Durante los siguientes cuatro cursos académicos se implementó la experiencia educativa con la intención de poder contrastar los resultados obtenidos y valorar la relevancia real de los conceptos ya examinados.

El estudio realizado se encuentra enmarcada en el módulo II: Dirección y gestión de Centros Gerontológicos en el tema 13: Software social y estrategias para el envejecimiento activo (Figura 2).

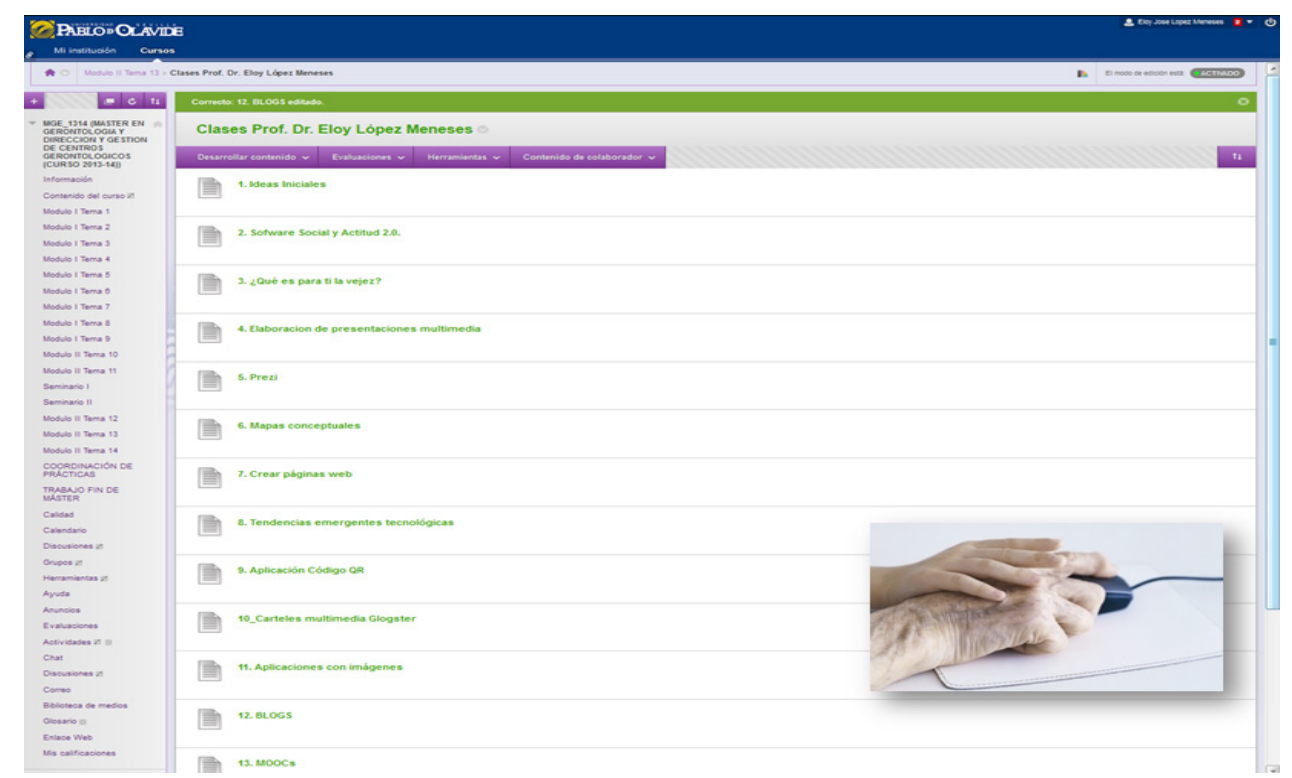

Figura 2. Organigrama del Tema 13 (extraído de la plataforma blackboard Learning UPO).

La investigación educativa consistía en plantear al estudiantado una reflexión introspectiva a través de nubes de conceptos sobre los principales aspectos que caracterizan el concepto de "vejez", con la aplicación social Wordle (http://www. wordle.net) durante los cursos 2012-13 y 2013-14 y la herramienta Tagxedo (http:// www.tagxedo.com/) en los cursos 2014-15 y 2015-16. Este software social permite 
diseñar nubes de etiquetas ("tag clouds") de forma dinámica y fácil a partir de los conceptos que se le proporcionan.

En la experiencia de postgrado los estudiantes deben indicar los 4 ó 5 conceptos que para ellos resultan más representativos de la Vejez. Posteriormente, ellos enviaban el enlace de la nube de conceptos con un breve comentario al correo electrónico del docente y se diseña un espacio web para difundirlo con la aplicación gratuita Jimdo: http://mayores3000.jimdo.com/ (Figura 3) que servía de repositorio al estudiantado para después realizar un debate reflexivo de las diferentes opiniones vertidas por el estudiantado de cursos anteriores.

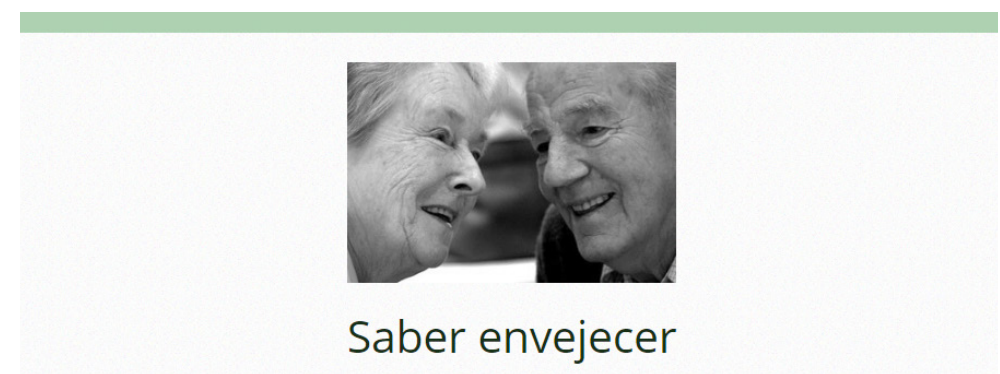

Curso 2015-16 Curso 2014-15 Curso 2013-14 Cursos 2012-13

Rúbrica/ Matriz de valoración

¿Qué significado tiene para ti la VEJEZ?

Reflexiona sobre las personas mayores y construye una "nube de cinco palabras" que expresen tu consideracion sobre ellas, con la Tagxedo (htip//wwwtagxedo:com/ )

Copia, por último, el enlace de la "nube de palabras" que te proporcionará la aplicación Wordle y envíalo al siguiente correo electrónico: eloy3000@gmail com, incluyendo en el mismo tu nombre, apellidos, y un comentario reflexivo y crítico que no supere las 400 palabras.
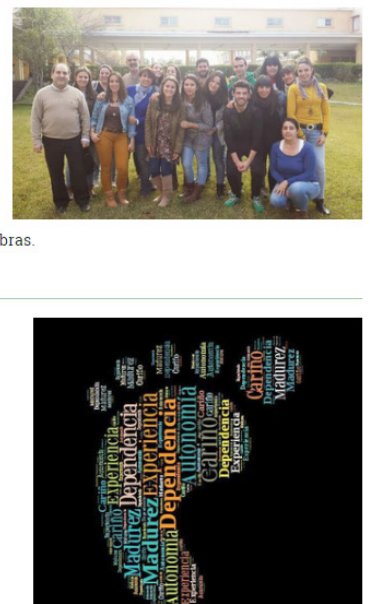

Figura 3. Espacio virtual de la experiencia innovadora universitaria. http://mayores3000.jimdo.com/

También, se diseñó una rúbrica para valorar la e-actividad (figura 4). 


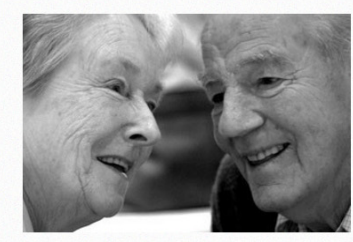

\section{Saber envejecer}

\section{Rúbrica de la e-actividad}

\begin{tabular}{|l|c|}
\hline \multicolumn{1}{|c|}{ CRITERIOS DE EVALUACION } & VALOR \\
\hline COMENTARIOS & 7 PUNTOS \\
\hline La nube de palabras ayuda a la reflexión social y educativa. & 2 \\
\hline $\begin{array}{l}\text { Los comentarios personales sobre la vejez son relevantes, } \\
\text { coherentes y significativos. }\end{array}$ & 4 \\
\hline Muestra un desarrollo lógico de las ideas. & 1 \\
\hline PRESENTACIÓN Word Clouds & 3 PUNTOS \\
\hline \begin{tabular}{l} 
Redacción clara y concisa. \\
\hline $\begin{array}{l}\text { Léxico amplio, adecuado, pertinente con una correcta } \\
\text { utilización de las normas ortográficas y de acentuación. }\end{array}$
\end{tabular} \\
\hline \begin{tabular}{l} 
Usabilidad del entomo Word Clouds. \\
\hline
\end{tabular} \\
\hline
\end{tabular}

Figura 4. Matriz de valoración de la e-actividad http://mayores3000.jimdo.com/rúbrica-matriz-de-valoración/

Por último, sirva a modo de ejemplificación el concepto y la presentación realizada por un estudiante del Máster de Gerontología y Dirección y Gestión de Centros Gerontológicos que se realizó en marzo de 2014 con la aplicación de software social denominada Wordle (Figura 5). Responsabilidad en el apoyo a la familia, Ocio en el disfrute de tiempo libre, Descanso en la evasión del estrés laboral, Participación en la realización de actividades de diversa índole, Sabiduría fruto de la experiencia vivida.

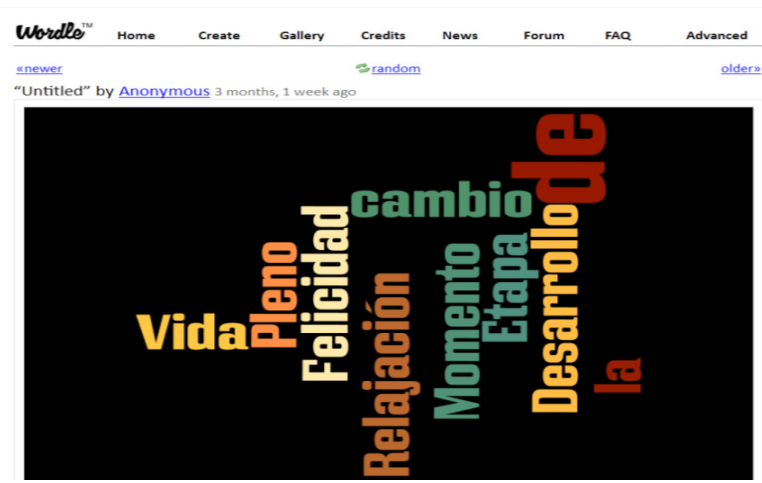

Figura 5. Aportación de estudiante (A) de la experiencia innovadora universitaria. Máster de Gerontología (UPO). 
O bien el trabajo realizado el 3 de marzo de 2016 con nubes de palabras por el estudiante Sergio García López (Figura 6).
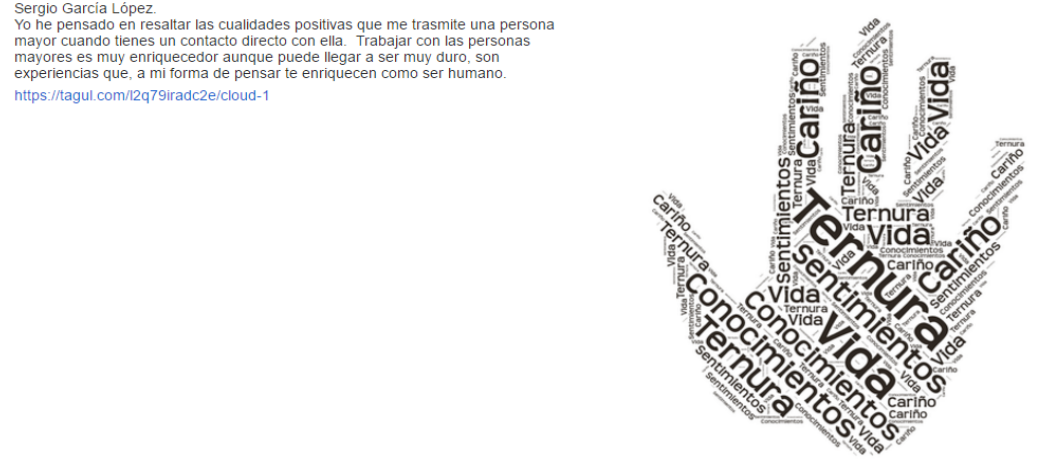

Figura 6. Aportación de estudiante (b) de la experiencia innovadora universitaria. Máster de Gerontología (UPO).

A continuación, se muestra las intenciones didácticas, el desarrollo de la investigación y los resultados más relevantes alcanzados durante el desarrollo de los cuatro cursos académicos en el Máster Oficial de Gerontología y Dirección y Gestión de Centros Gerontológicos.

\section{OBJETIVOS}

Los principales objetivos de la experiencia innovadora llevada a cabo fueron los siguientes: (1) Reflexión introspectiva sobre la concepción de la vejez y en el envejecimiento activo; (2) Fomentar el papel activo y autónomo de los estudiantes en el proceso de construcción del conocimiento; (3) Favorecer el aprendizaje multimodal entre los estudiantes; (4) Diseñar y elaborar nubes de ideas previas sobre las Concepciones Relacionadas con el proceso de Envejecimiento; (5) Fomentar el uso de software social; (6) Elaborar un repositorio de concepciones previas en relación con la Vejez.

El objetivo prioritario que se pretendía con esta investigación diacrónica lo largo de cuatro cursos académicos era comprobar la prevalencia de los conceptos de Vejez incorporados al repositorio a lo largo del periodo de tiempo estudiado (curso académico 12-13 al curso académico 15-16).

\section{METODOLOGÍA DE LA INVESTIGACIÓN}

Se utiliza como eje vertebrador metodológico la investigación-acción colaborativa virtual por parte del estudiante universitario como una manera de 
fomentar sus propias competencias genéricas y específicas en el Espacio Europeo de Educación Superior (Pool-Cibrian y Martínez-Guerrero, 2013; Zimmerman y Schunk, 2011). Esta concepción metodológica se fundamenta en la interdependencia positiva que fomenta la responsabilidad grupal del trabajo colaborativo (Kitsantas y Dabbagh, 2010; Linnenbrink y Pintrich, 2003). A su vez, las experiencias aportadas por Gómez-Lucas y Álvarez-Teruel (2011) y Millis y Rhem (2010), evidencian que al emplear el trabajo colaborativo como una estrategia en el aula universitaria éste contribuye a potenciar el aprendizaje, al permitir la confrontación de puntos de vista y opiniones, ayuda a revalorizar la perspectiva propia y facilita el intercambio con el otro, pues activa y conduce al aprendizaje para abordar con éxito situaciones comunicativas entre iguales.

Para el presente estudio analítico se revisaron las aportaciones efectuadas por el grupo estudiante analizando las palabras o conjuntos de significados como unidades de registro. Posteriormente se transcribió y categorizó la trama conceptual tomando como marco de referencia las pautas establecidas por diferentes autores (Bogdan y Biklen, 1992; Miles y Huberman, 1994).

En la fase primera se realizó el filtrado en bruto de los textos y nube de palabras multimedia por medio de la técnica de "reducción de datos". Esta fase constituye la realización de procedimientos racionales que consisten en la categorización y codificación de los datos, identificando y diferenciando unidades de significado. En primer lugar, se procedió a la "categorización de los datos". Esta categorización implica la simplificación y selección de información para hacerla más manejable. Este proceso se estructuró en dos subfases: "Separación de unidades": consiste en separar segmentos de información siguiendo algún tipo de criterio como puede ser espacial, temporal, temático, gramatical. "Identificación y clasificación de unidades": consiste en clasificar conceptualmente las unidades que son cubiertas por un mismo tópico con significado. El procedimiento puede ser inductivo, es decir, a medida que se van examinando los datos, o deductivo, habiendo establecido previamente el sistema de categorías sobre el que se va a categorizar, tras la revisión de literatura específica sobre la temática objeto de estudio. En este estudio optamos por una clasificación mixta por medio de la técnica de "síntesis y agrupamiento". Esta fase está unida realmente a la anterior dado que la propia categorización implica la síntesis. Esta fase también estuvo presente una vez que ha concluido el proceso de categorización y algunas categorías se agrupan en metacategorías.

Una vez finalizado el proceso de "codificación", se asignó una categoría a cada unidad textual. En este sentido, cada unidad seleccionada ha sido codificada para su recuento frecuencial, mediante el programa de análisis estadístico SPSS Statistics 20.0 (Castañeda, Cabrera, Navarro, y Vries, 2010).

En la fase segunda se realizaron los procesos de "interpretación e inferencia". El software SPSS facilita la creación de archivos de datos de forma estructurada y 
organizar las bases de datos para que puedan ser analizadas con diversas técnicas estadísticas. (Figura 5).

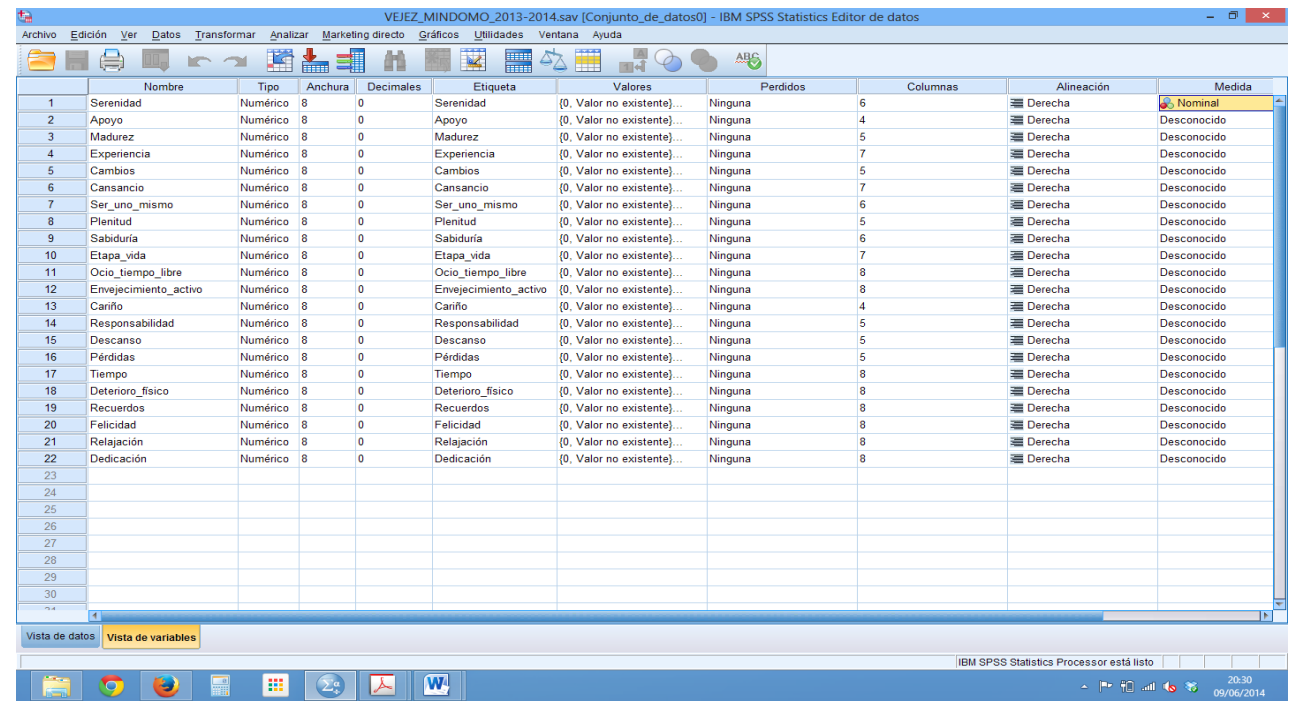

Figura 5. Sistema categorial elaborado con IBM SPSS Statistics 20.0

Para finalizar el proceso de análisis de los conceptos y dado que era interesante conocer la prevalencia de los conceptos sobre vejez a lo largo del estudio diacrónico, se efectuó un análisis estadístico a partir de Tablas de Contingencia y Resumen de procesamiento de casos y así obtener los conceptos que han perdurado en el tiempo junto con el peso específico que han tenido cada uno de ellos. Por último, se tuvo en consideración, para valorar de modo positivo la prevalencia del concepto, que al menos apareciera en tres de los cuatro cursos académicos estudiados.

En el siguiente apartado se muestran los resultados, conclusiones y limitaciones de la investigación realizada.

\section{RESULTADOS}

Los estudiantes del Máster de Gerontología y Dirección y Gestión de Centros Gerontológicos de la Universidad Pablo de Olavide, en los distintos cursos escolares, fueron cumpliendo de manera satisfactoria los objetivos marcados en el estudio, reflejando en las nubes de palabras lo que ellos/as entendían como el significado del concepto de "vejez" partiendo de su reflexión introspectiva sobre la concepción de la vejez y en el envejecimiento activo. Por otro lado la metodología desarrollada ha fomentado en todo momento el papel activo y autónomo de los estudiantes en el 
proceso de construcción del conocimiento, consiguiendo alcanzar un aprendizaje multimodal entre los estudiantes y el uso de software social. Por último se ha ido construyendo un repositorio colectivo de concepciones previas en relación con la Vejez.

Para exponer los resultados obtenidos a lo largo del estudio diacrónico, primero vamos a exponer los resultados obtenidos para cada uno de los cursos, para posteriormente realizar una valoración sobre aquellos conceptos que prevalecen a lo largo del tiempo.

Respecto al curso escolar 2012-2013, en la gráfica 1, se expresan los conceptos que se asocian a la Vejez.

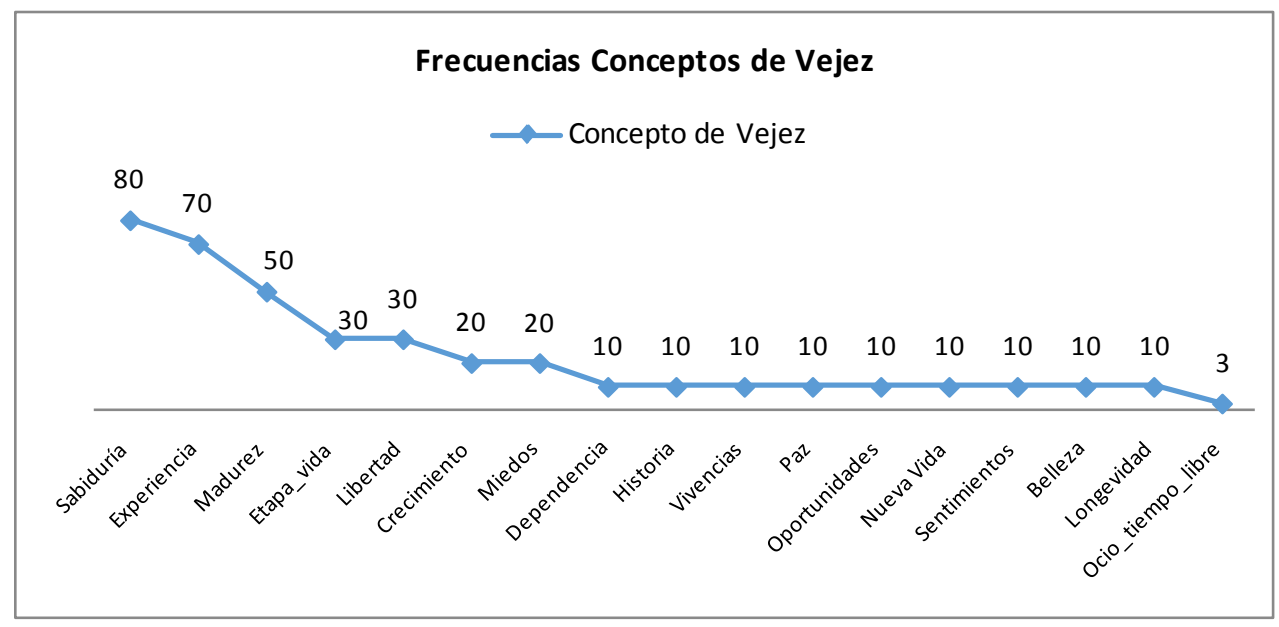

Gráfica 1. Porcentajes de aparición de los conceptos asociados al significado de vejez para estudiantes del curso 2012-2013 del Máster de Gerontología. Universidad Pablo de Olavide.

En este sentido, se constata en la gráfica anterior que el concepto más representativo asociado a la vejez son la "Sabiduría" con el (80.0\%) y la "Experiencia" (70.0\%). Seguidamente, el concepto de "Madurez" con el (50.0\%). Por último, aparecen otra serie de acepciones con una menor prevalencia, pero que visualiza el abanico léxico-semántico inherente a la idea de vejez que infiere el propio estudiantado de posgrado, como pueden ser "Etapa de la vida" y "Libertad" con un (30.0\%) o "Crecimiento y miedos" con un (20.0\%). Por último, otros conceptos que surgen en la dinámica activa del aula son "Dependencia", "Vivencias", "Belleza".

Durante el curso escolar 2013-2014, se expresan los conceptos recopilados en la gráfica 2. 


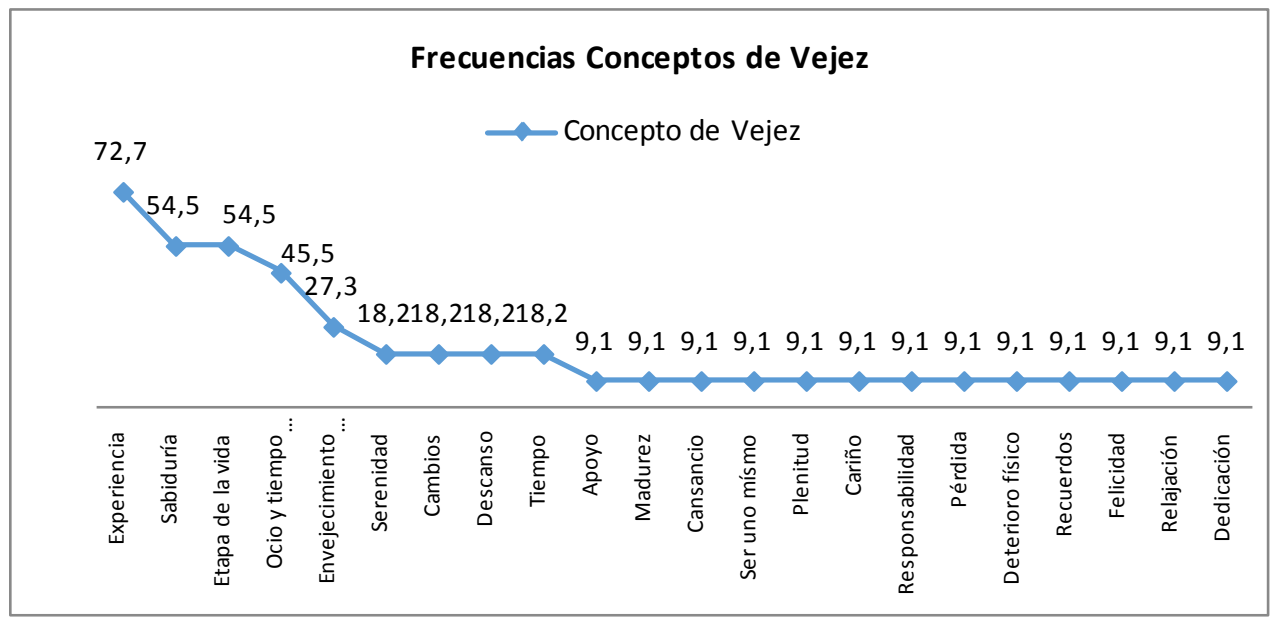

Gráfica 2. Porcentajes de aparición de los conceptos asociados al significado de vejez para estudiantes del curso 2013-2014 del Máster de Gerontología. Universidad Pablo de Olavide.

En este curso académico, el concepto que brilla con luz propia y sirve como epicentro globalizador al concepto de Vejez es la "Experiencia" con un $(72.7 \%)$, seguido de los conceptos de "Sabiduría y Etapa de la vida" (54.5\%), "Ocio y tiempo libre" (45.5\%), "Envejecimiento activo" (27.3\%) con una mayor diferenciación sobre el resto de respuestas reflejadas.

Otras características que los estudiantes han vinculado al proceso de Envejecer son: Ser una fase colmada de "Serenidad", "Cambios físicos" importantes que pueden modificar el estilo de vida, "Descanso" dado que se termina la vida laboral activa y "Tiempo" para disfrutar de la familia y amistades con un $18,2 \%$ y ser un pilar de "Apoyo", "Responsabilidad" y "Dedicación" para cuidar y preocuparse, luchar y sacar adelante a la familia, sobre todo en estos momentos de crisis, "Madurez personal" después de toda una vida, "Cansancio" tras muchas batallas vividas a lo largo de la existencia, "Ser uno mismo", "Plenitud de la vida", en la que se han conseguido cosas, "Cariño" hacia la familia y amistades, etapa de "Pérdidas" dado que familiares directos y amistades pueden fallecer, "Deterioro físico", "Recuerdos de toda la vida", de las experiencias vividas, "Felicidad" dado que pueden disfrutar de tiempo para hacer actividades que les gusten y por último de "Relajación" con un $9.1 \%$.

Referente al estudiantado correspondiente al curso académico 2014-2015, valora como concepto central que emana de la acepción de la vejez la "Autonomía" con una prevalencia del $81.8 \%$, como se indica en la gráfica 3. Es un concepto inherente a la idea de vejez como una etapa de la vida positiva y llena de vitalidad y de posibilidades vitales. El concepto que le sigue, con un 54.2\% es la "Sabiduría", concepto que ya ha sido mencionado por el alumnado de cursos anteriores. Con 
menor relevancia se vislumbran los conceptos de "Madurez", "Cariño", con un 18.2\%, todos ellos presentan una visión también positiva

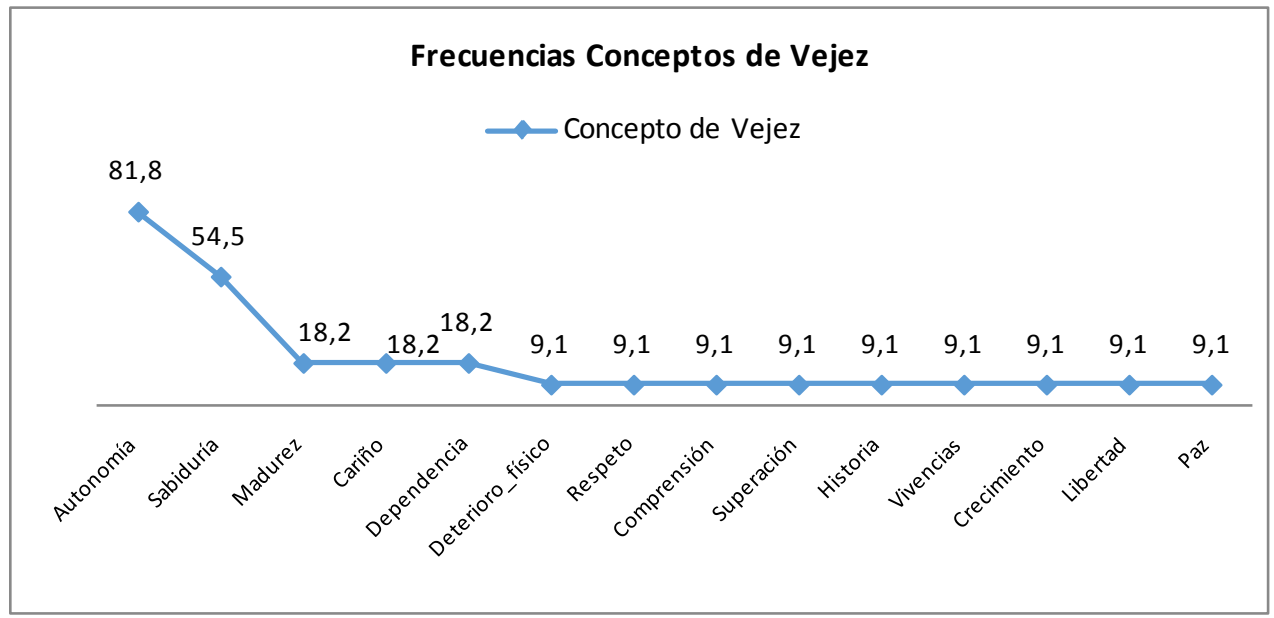

Gráfica 3. Porcentajes de aparición de los conceptos asociados al significado de vejez para estudiantes del curso 2014-2015 del Máster de Gerontología. Universidad Pablo de Olavide.

En la gráfica 4 se muestran los conceptos definitorios de la etapa de la vejez correspondiente al curso académico 2015-16.

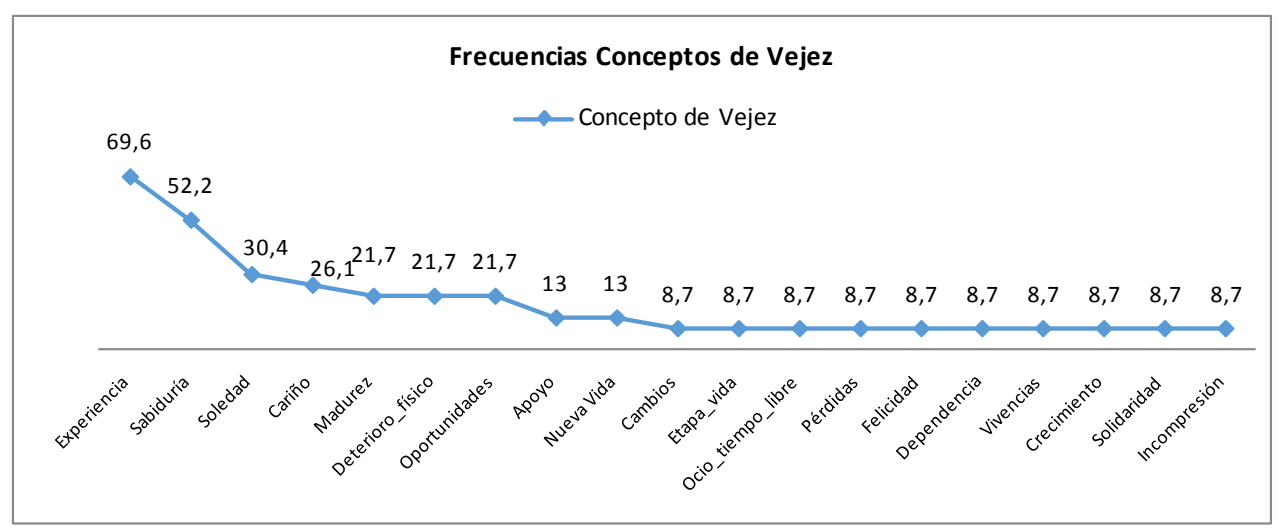

Gráfica 4. Porcentajes de aparición de los conceptos asociados al significado de vejez para estudiantes del curso 2015-2016 del Máster de Gerontología. Universidad Pablo de Olavide.

Cabe mencionarse que en el curso académico 2014-15, el concepto que de modo reiterado se manifiesta es la "Experiencia", con un (69.6\%). Posteriormente, 
el concepto de "Sabiduría", con un (52.2\%). Ambas acepciones revelan una imagen de la senectud como una etapa que confiere a la persona un bagaje experiencial muy importante logrado a lo largo de la vida. En contraposición, cabe resaltar con una prevalencia del $30.4 \%$ la "Soledad", que se encuentra orientado a aspectos negativos. Otros conceptos que surgen con un porcentaje de $21.7 \%$ son los de "Madurez", "Deterioro físico" y "Oportunidades".

Una vez presentados los resultados por curso académico, a continuación, se expone la prevalencia de los conceptos sobre la "Vejez" a lo largo del tiempo, para observar su oscilación. Y como se indicó anteriormente, se valora un concepto prevalente en el tiempo si aparecería en tres de los cuatro cursos académicos estudiados, así en la gráfica 5 se representan los datos recogidos.

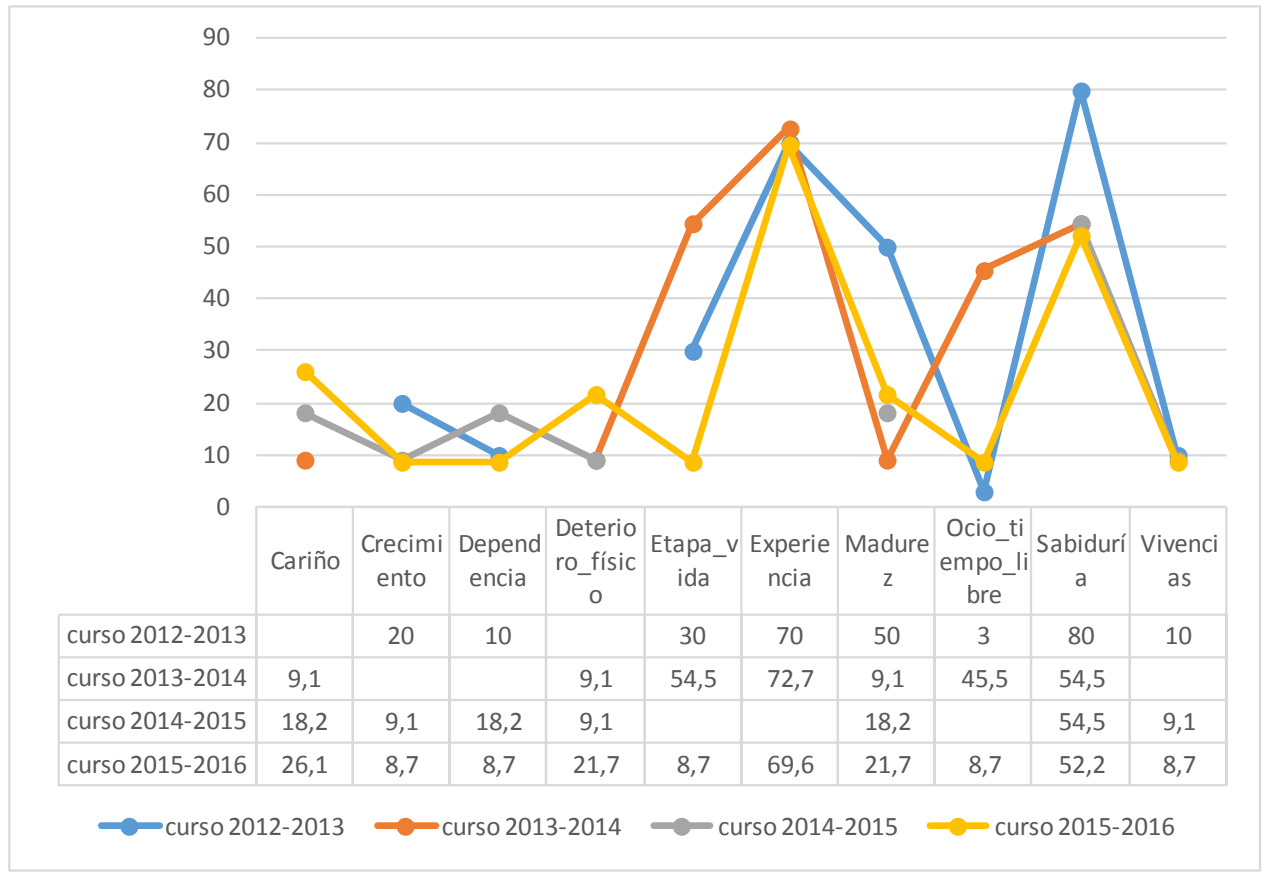

Gráfica 5. Comparativa de la prevalencia de los conceptos sobre vejez para estudiantes de los cursos académicos 2012-2013; 2013-2014; 2014-2015 y 20152016 del Máster universitario en Gerontología y Dirección y Gestión de Centros Gerontológicos.

Al realizar una comparativa entre la prevalencia de los conceptos relevantes para el grupo de estudiantes, se observa una predominancia de dos conceptos que emergen del estudio diacrónico. Dichos conceptos son los de "Sabiduría", teniendo una representatividad muy alta entre el $80.0 \%$ y el $52.2 \%$ en todos los cursos. También destaca el concepto "Experiencia", con unos porcentajes de aparición 
de entre el $69.6 \%$ y el $72.7 \%$ que aunque en uno de los cursos académicos no se manifiesta, en el resto de ellos posee una alta prevalencia. El de "Madurez", con porcentaje con una mayor variabilidad (entre un $9.1 \%$ a un $50.0 \%$ ), también resalta por su alta representatividad. En simbiosis con estos conceptos emanan otros como el "Cariño", con un porcentaje máximo de utilización del 26.1\%, el ser una "Etapa de Crecimiento", con un porcentaje inicial del $20.0 \%$ que ha disminuido en los últimos cursos académicos; una "Etapa de la Vida", un periodo vital en la se permite un mayor acceso al "Ocio y el Tiempo Libre" y en la que se acumulan una serie de "Vivencias". Por último, destacar que los conceptos mencionados evocan positividad hacia esta etapa en la vida de las personas, aun así, también aparecen algunos conceptos que se podría denominar menos positivos, como son la "Dependencia" o el "Deterior Físico", pero se puede entender que son también conceptos inherentes a la propia naturaleza humana y al desarrollo vital de las personas.

\section{GONCLUSIONES}

Entre las principales conclusiones que se obtienen, cabe mencionar que los objetivos planteados en la experiencia de posgrado: reflexionar y analizar a través de nubes de conceptos, la acepción de la vejez, favorecer el desarrollo de actitudes críticas y conocer una aplicación 2.0 (Wordle y tagxedo) se han conseguido plenamente, como se observan en los trabajos realizados por el alumnado de posgrado en el espacio virtual http://mayores3000.jimdo.com/

También es de resaltar que el grupo de estudiantes ha relacionado el proceso del envejecimiento con la sabiduría en concordancia con otros autores (Levy, 1996; Isaacowitz y Seligman, 2001; Mikulak, 2012), o con aspectos vinculados a la experiencia y bagaje experiencial (Carbajo Vélez, 2009), un periodo que prevalecen los miedos como apunta Viguera (2000) o bien, una etapa llena de emociones y nuevas oportunidades (Steverink, et al., 2000; Gómez Lucas. y Álvarez Teruel, 2011 Stephan, et al., 2013; Sarasola, López Meneses, y Fernández, 2013).

Asimismo, desde una visión negativa define la vejez como un estado deficitario, donde se producen pérdidas significativas e irreversibles (Corraliza, 2000). En este sentido, se describe a la persona mayor como físicamente disminuido y con deterioro físico en concordancia con Matras (1990). Por otra parte, los estudiantes valoran muy positivamente el software social para la elaboración de nubes de conceptos: "Wordle" (URL: http://www.wordle.net/) y tagxedo (http://www.tagxedo.com/) por ser intuitivos, fáciles de manejar y funcionales, fruto del debate realizado en la última parte de la sesión formativa.

Consideramos, globalmente, que estos estudios además de su valor científico contribuyen a dar respuesta a un gran problema social que resulta fundamental en el bienestar de un país, y es la atención a las personas mayores. Por supuesto han jugando y seguirán jugando un papel decisivo en la sociedad. En este campo 
resulta fundamental no sólo del análisis científico de una compleja problemática, sino asimismo su aplicación en el ámbito de políticas sociales que contribuyan a mejorar la calidad de vida de los ciudadanos (Ponce, Gómez Galán y Pagán, 2017 y 2018). En última instancia, por supuesto, permitirá avivar el pensamiento divergente y la creatividad humana que, como indica Martínez-Migueléz (2011), hace que la información esté ahí, el ambiente esté lleno de ella, pero realmente lo que hay que hacer es aprender a jugar con sus elementos, desarrollando la creatividad y la originalidad con estas metas.

\section{BIBLIOGRAFÍA}

Abellán García, A. y Ayala García, A. (2012). Un Perfil de las Personas Mayores en España, 2012. Indicadores Estadísticos Básicos. Recuperado de: http://www. imsersomayores.csic.es/documentos/documentos/pm-indicadoresbasicos12.pdf

Aguaded, J. I., López Meneses, E y Alonso, L. (2010). Innovating with Blogs in University Courses: a Qualitative Study. The New Educational Review, 22 (3-4), 103-115.

Aguaded, J. I., López Meneses, E. y Jaén, A. (2013). Portafolios Electrónicos Universitarios para una Nueva Metodología de Enseñanza Superior. Desarrollo de un Material Educativo Multimedia (MEM). Revista de Universidad y Sociedad del Conocimiento (RUSC), 10 (1), 7-28.

Agudo, S.; Pascual, M. A. y Fombona, J. (2012). Usos de las Herramientas Digitales entre las Personas Mayores. Comunicar, 39, 193-201. doi: 10.3916/c39-2012-03-10

Amador-Muñoz, L y Esteban-Ibáñez, M. (2015). Calidad de vida y formación en hábitos saludables en la alimentación de personas mayores. Revista de Humanidades, 25. Recuperado de: http://revistas.uned.es/index.php/rdh/article/view/14374/13807

Bazo, M.T. (1990). La Sociedad Anciana. Madrid: CIS-Siglo XXI.

Beauvoir de, S. (1983). La Vejez. Edhasa: Barcelona.

Bogdan, R. y Biklen, S. K. (1992). Investigación Cualitativa de la Educación. Needham Heights, MA: Allyn and Bacon.

Carbajo Vélez, M. C. (2009). Mitos y Estereotipos sobre la Vejez. Propuesta de una Concepción Realista y Tolerante. Revista Ensayos, Revista de la Facultad de Educación de Albacete, 24, 87-96.

Castañeda, M. B., Cabrera, A. F. Navarro, Y. y Vries, W. (2010). Procesamiento de Datos y Análisis Estadísticos Utilizando SPSS: Un Libro Práctico para Investigadores y Administradores Educativos. Porto Alegre: EDIPUCRS.

Cook, J. (2013). Senior Adult Learners at University: Age is Just a Number [Tesis Doctoral], The University of Queensland, Herston (Australia).

Corraliza, J. A. (2000). Vejez y Sociedad. En R. Fernández-Ballesteros (Dir.). Gerontología Social (pp. 229-239). Madrid: Pirámide. 
Cróquer, E y Paz, J. L. (2013). Pensar (en) la Universidad. Una Reflexión en Torno al Sentido de la Universidad y su Razón de Ser en el Presente Mundializado. Argos, 30 (59), 47-59. Elosúa, M. T. (2002). El Desarrollo de la Memoria en la Vida Adulta y en la Vejez. En VV.AA. Psicología Evolutiva II. Desarrollo Cognitivo y Lingüístico (pp. 299-328). Madrid: UNED.

Escarbajal de Haro, A. (2003). Personas Mayores, Educación y Aprendizaje. En J. Sáez, (Coord.) Educación y Aprendizaje en las Personas Mayores (pp. 159-181). Madrid: Dykinson, S.L.

European Union. (2015). The 2015 Ageing Report. Economic and Budgetary Projections for the 28 EU Member States. Luxembourg: Publications Office of the European Union.

Fernández-Ballesteros, R. (2000). Gerontología Social. Madrid: Ediciones Pirámide.

Fernández-Ballesteros, R., Zamarrón M.D., López, M.D., Molina, M.A., Díez, J., Montero, P. y Schettini, R. (2010). Envejecimiento con Éxito: Criterios y Predictores. Psicothema, 22 (4), 641-647.

Gairín, J., Rodríguez-Gómez, D., Armengol, C., y del Arco Bravo, I. (2014). El Acceso a la Universidad para Personas Mayores en España. Los Programas Universitarios para Personas Mayores: Revisando la Realidad. Revista de Orientación Educacional, 27(51), 45-66.

Gallardo, C., Peñacoba, C. y Martínez, A. (2009). Las Habilidades para la Vida como Modelo de una Experiencia de Innovación Docente en la Universidad Rey Juan Carlos. En VV.AA. I Encuentro de Intercambio de Experiencias en Innovación Docente en la Universidad Rey Juan Carlos, Madrid: Universidad Rey Juan Carlos.

García Rodríguez, M. P., Meseguer Martínez, L., González Losada, S., \& Pozo Muñoz, C. (2016). ¿Cómo Acceden los Mayores a la Universidad en España? Nuevas Oportunidades para la Formación a lo Largo de la Vida. Revista Española de Pedagogía, 253, 515-535.

Gómez Galán, J. \& Lacerda, G. (Eds.) (2012). Informática e Telemática na Educação. Vol. I. As Tecnologias de Informação e Comunicação na Educação. Brasilia: Liber Livro Editora/Universidade de Brasilia

Gómez Lucas, M. C. y Álvarez Teruel, J. D. (Coords.) (2011). El trabajo Colaborativo como Indicador de Calidad del Espacio Europeo de Educación Superior. Alcoy: Marfil.

González-Raimundí, Z. (2015). A Phenomenological Study of Adult College Student Experience in Nontraditional Education Programmes. International Journal of Educational Excellence, 1(1), 43-75. doi:10.18562/ijee.2015.0002

González-Raimundí, Z. (2015). University Educational Opportunities to Adults 50 Years of Age or Older: Fundamental Reasons and Characteristics. International Journal of Educational Excellence, 2(2), 65-76. doi:10.18562/ijee.019 
Isaacowitz, D.M. y Seligman, M.E.P. (2001). Is Pessimism a Risk Factor for Depressive Mood among Community-dwelling Older Adults? Behaviour Research and Therapy, 39, 255-272. doi: 10.1016/s0005-7967(99)00178-3

Kasworm, C. E. (2012). US Adult Higher Education: One Context of Lifelong Learning. International Journal of Continuing Education and Lifelong Learning, 5 (1), 1-19.

Kitsantas, A. y Dabbagh, N. (2010). Learning to Learn with Integrative Learning Technologies (ILT): A Practical Guide for Academic Success. Estados Unidos: Information Age Publishing.

Levy, B. (1996). Improving Memory in Old Age Through Implicit Self-Stereotyping. Journal of fereonality and Social Psychology, 71(6), 1092-1107.

Linnenbrink, E. y Pintrich, P. (2003). The Role of Self-Efficacy beliefs in Student Engagement and Learning in the Classroom. Reading and Writing Quarterly, 19, 119-137.

López Meneses, E. y Gómez Galán, J. (2010). Prácticas Universitarias Constructivistas e Investigadoras con Software Social. Praxis, 5, 23-45.

Markle, G. (2015). Factors Influencing Persistence among Nontraditional University Students. Adult Education Quarterly, 65 (3), 267-285. doi: 10.1177/0741713615583085

Martin García, A. V. y Requejo, A. (2005). Fundamentos y Propuestas de la Educación no Formal con Personas Mayores. Revista de Educación, 338, 45-66.

Martínez-Miguélez, M. (2011). La Docencia Universitaria Hoy: Metas, Técnicas y Desafíos. Argos, 28 (55), 108-124.

Matras, J. (1990). Dependency, Obligations and Entitlement: A New Sociology of Aging. Englewood Cliffs, N. J.: Prentice-Hall.

Mikulak, A (2012). Aging and Wisdom: Culture Matters. APS Journal Psychological Science. Recuperado de: http://www.psychologicalscience.org/index.php/news/ releases/does-wisdom-really-come-with-age-it-depends-on-the-culture.html

Miles, M. B. y Huberman, A. (1994). Qualitative Data Analysis: An Expanded Sourcebook. Newbury Park, CA: Sage.

Millis, B.J. y Rhem, J. (2010). Cooperative Learning in Higher Education. Across the Disciplines, across the Academy. Virginia: Stylus Publishing.

Montañés Rodríguez, J. y Latorre Postigo, J. M. (2004). Psicología de la Vejez. Estereotipos Juveniles sobre el Envejecimiento. Cuenca: Ediciones de la Universidad de Castilla-La Mancha.

Montero, I. (2005). El Interés de las Personas Mayores por la Educación: Qué Educación. Estudio de Campo [Tesis Doctoral]. Editorial Universidad de Granada. Departamento de Pedagogía. Granada (España).

Organización Mundial de la Salud (2002). Envejecimiento Activo: Un Marco Político. Revista Española de Geriatría y Gerontología, 37, 74-105. 
Organización Mundial de la Salud (2015). Informe Mundial sobre el Envejecimiento y la Salud. EEUU (impression): Organización Mundial de la salud. Recuperado de: http://apps.who.int/iris/bitstream/10665/186466/1/9789240694873_spa.pdf?ua=1

Organización Panamericana de la Salud. Oficina Regional de la Organización Mundial de la Salud. (OPS). (2002). Promover un Estilo de Vida para las Personas Mayores. En VV.AA. Guía Regional para la Promoción de la Actividad Física. Washington.

Ponce O., Gómez Galán, J. y Pagán, N. (2017). Philosophy of Science and Educational Research: Strategies for Scientific Effectiveness and Improvement of the Education. European Journal of Science and Theology, 13 (3), 137-147.

Ponce O., Pagán, N. y Gómez Galán, J. (2017). Research of Educational Policies: Science over Ideology, Espacios, 39 (43), 1-8.

Pool-Cibrian, W. J. y Martínez-Guerrero J. I. (2013). Autoeficacia y Uso de Estrategias para el Aprendizaje Autorregulado en Estudiantes Universitarios. Revista Electrónica de Investigación Educativa, 15 (3), 21-37.

Rodríguez-Rodríguez, V., Rodríguez-Mañas, L., Sancho-Castiello, M. y Díaz-Martín, R. (2012). Envejecimiento. La Investigación en España y Europa. Revista Española de Geriatría y Gerontología, 47 (4), 174-179.

Rojo-Pérez, F., Fernández-Mayoralas, G., Forjaz, M. J., Delgado-Sanz, M. C., AhmedMohamed, K., Martínez-Martín, P., Prieto-Flores, M. E. y Rojo-Abuín, J. M. (2009). Población mayor, Calidad de Vida y Redes de Apoyo: Demanda y Prestación de Cuidados en el Seno Familiar. Madrid: Escuela Nacional de Sanidad. Instituto de Salud Carlos III. Ministerio de Ciencia e Innovación. Recuperado de: http://www. isciii.es/htdocs/publicaciones/documentos/Poblacion_mayor.pdf

Sáez, J. (2002). Hacia la Educación Intergeneracional. Concepto y Posibilidades. En Sáez (Coord.). Pedagogía Social y Programas Intergeneracionales: Educación de Personas Mayores (pp. 99-112). Málaga: Ediciones Aljibe S.L.

Sarasola, J. L., López Meneses, E. y Fernández, E. (2013). Experiencias de Posgrado sobre el Envejecimiento a través de Nubes de Palabras. Revista Didáctica, Innovación y Multimedia, 25, 1-13.

Schuetze, H. y Slowey, M. (2002). Participation and Exclusion: A Comparative Analysis of Non-Traditional Students and Lifelong Learners in Higher Education. Higher Education, 44, 309-32. doi: 10.1023/a:1019898114335

Stephan, Y., Chalabaev, A., Kotter-Grühn, D. y Jaconelli, A. (2013). Feeling Younger, Being Stronger: An Experimental Study of Subjective Age and Physical Functioning Among Older Adults. Journal of Gerontology: Psychological Sciences, 68, 1-7. doi: $10.1093 /$ geronb/gbs037

Steverink, N. Westerhof, G.J., Bode, C. y Dittmann-Kohli, F. (2000). The Personal Experience of Aging, Individual Resources, and Subjective Well-Being. The Journals of Gerontology, Series B. Psychological Sciences and Social Sciences, $56,364-373$. 
Thumala, D., Arnold, M., y Urquiza, A. (2010). Opiniones, expectativas y evaluaciones sobre diferentes modalidades de inclusión/exclusión social. Argos 27 (53), 91-122

UNFPA y Help Age Internacional (2012). Aging in the Twenty-First Century: A Celebration and a Challenge. UNFPA y HelpA ge International.

Viguera, V. (2000). Identidad y Autoestima en los Adultos Mayores. Revista Tiempo. El Portal de la Psicogerontología, 5. Recuperado de: http://www.psicomundo.com/ tiempo/tiempo5.htm

VV.AA (2015). Estimaciones y Proyecciones de Población a Largo Plazo 1950-2100. CELADE/ CEPAL, Observatorio Demográfico. Recuperado de: http://www.cepal. org/es/estimaciones-proyecciones-poblacion-largo-plazo-1950-2100

Yanguas, J. J. (2006). Análisis de la Calidad de Vida Relacionada con la Salud en la Vejez desde una Perspectiva Muntidimensional. IMSERSO. Madrid: Ministerio de Trabajo y Asuntos Sociales. Recuperado de: http://envejecimiento.csic.es/ documentos/documentos/yanguas-analisis-01.pdf

Zamarrón, M ${ }^{a}$ D. (2013). Envejecimiento Activo: Un Reto Individual y Social. Sociedad y Utopía. Revista de Ciencias Sociales, 41, 449-463.

Zimmerman, B.J. y Schunk, D. (2011). Self-Regulated Learning and Performance an Introduction and an Overview. En B. J. Zimmerman y D. Schunk (Eds.). Handbook of Self-Regulation of Learning and Performance. England: Routledge. 